\title{
Giant Cerebriform Melanocytic Nevus of the Scalp; A Case report
}

fatemeh mohaghegh ${ }^{1}$, ali asilian ${ }^{2}$, Samin Nabavinejad ${ }^{1}$, and Mina Rezaei ${ }^{3}$

${ }^{1}$ Affiliation not available

${ }^{2}$ Skin Disease Research Center and Department of Dermatology, Isfahan University School

of Medicine, Isfaha, Iran

${ }^{3}$ Isfahan University of Medical Sciences

May 27, 2020

\begin{abstract}
Giant congenital melanocytic nevi are brown-black lesions larger than $20 \mathrm{~cm}$ caused by benign proliferation of melanocytes. They either regress or progress in time in matters of thickness and size and may transform to malignancies. Here we report a twelve year old patient with giant melanocytic nevus of the scalp.
\end{abstract}

\section{Giant Cerebriform Melanocytic Nevus of the Scalp; A Case report}

Running title: Giant congenital melanocytic nevus

Fatemeh Mohaghegh (1), Ali Asilian (2), Samin Nabavinejad (3), Mina Rezaei (4)

1. Assistant professor of Dermatology, Department of Dermatology, Skin Diseases and Leishmaniasis Research Center, Isfahan University of Medical Sciences, Isfahan, Iran

2. Professor of Dermatology, Department of Dermatology, Skin Diseases and Leishmaniasis Research Censer, Isfahan University of Medical Sciences, Isfahan, Iran

3. Resident of Dermatology, Department of Dermatology, Skin Diseases and Leishmaniasis Research Center, Isfahan University of Medical Sciences, Isfahan, Iran

4. Medical Student, School of Medicine, Isfahan University of Medical Sciences, Isfahan, Iran

* Corresponding author : Samin Nabavinejad

Email: saminstrs@gmail.com

Address: Isfahan University of Medical Sciences, Isfahan, Iran

Postal code: 81746-73461

Phone number: 983136680048

Conflict of interest : None of the authors declare any conflicts of interest.

Consent : Written consent was taken from patient's guardians for publishing the case and viewing the pictures. This case report was approved by the bioethics committee of Isfahan University of Medical Sciences.

Key-words: Melanocytic nevus, giant congenital melanocytic nevi, malignant melanoma, neurocutaneous melanosis, congenital nevi

Key clinical Message 
Giant melanocytic nevus is a rare condition caused by benign proliferation of melanocytes. There is a slight risk of malignancy in these lesions which should be noticed especially when they become larger. GCMN can be removed by plastic surgery.

\section{Introduction}

Congenital melanocytic nevi are brown-black skin lesions caused by benign proliferation of melanocytes. They are mostly present at birth, however some authors consider melanocytic nevi developing within 2 years of life congenital as well (1). The incidence of CMN (congenital melanocytic nevi) is about 1\%. They are mostly sporadic, caused by somatic mutations, although familial cases have also been reported (2). CMN are categorized into three groups: small $(<1.5 \mathrm{~cm})$, medium $(1.5-20 \mathrm{~cm})$ and large or giant $\mathrm{CMN}(>20 \mathrm{~cm})$ (3).

There is a risk of malignant transformation both in prepubertal and post pubertal patients which is higher in larger lesions. The risk of transformation of GCMN(giant congenital melanocytic nevi) to melanoma is estimated between 0 to $3.8 \%(4,5)$. Another complication associated with GCMN is neurocutaneous melanosis (NCM), characterized by abnormal melanosis of the central nervous system (6).

Typical histopathological findings in CMN include; the presence of nevomelanocytes within the lower dermis, extension of nevomelanocytes between collagen bundles, around hair follicles, eccrine apparatus and sebaceous glands. CMN are often removed by surgery (7).

Here we report a 12-year old female immigrant from Afghanistan with multiple melanocytic nevi on the face and trunk and a giant congenital cerebriform melanocytic nevus on the scalp.

Case Report

A twelve year-old female immigrant from Afghanistan was admitted in the dermatology department of Alzahra hospital in 2020 with multiple brown lesions on the trunk and face and a large cerebriform plaque on the right side of her scalp.

She was born with multiple brown papules and nodules on the trunk and face and a brown patch on the right side of her scalp. She reported a small increase in the size of the lesions on her body and face over time and there was no associated pain or itching. According to her parents, the lesion on the scalp was a small brown flat patch at birth and had increased in size and thickness over the last 8 years and in the past 5 years it became nodular and cerebriform. The patient complained about intense itching and discharge which had recently become malodorous.

Her birth and developmental history were normal. The family history was negative for congenital nevi or melanoma and she was born of a non-consanguineous marriage. The patient had normal intelligence without any history of traumatic brain injury, dermatological disorder, endocrine disorder, or cancer and there were no similar cases in her family.

In physical examination, her developmental and nutritional status was normal. The head circumference was in normal range and her neurologic, cardiovascular, musculoskeletal and ophthalmic examinations didn't show any abnormalities.

On skin examination, there were multiple non-tender brown papules and nodules on her back, abdomen and face (Figure 1-a). There was no hypertrichosis or ulcer. The mass on the scalp sized $22 \mathrm{~cm} \times 18 \mathrm{~cm} \times 2.5$ $\mathrm{cm}$ approximately with a nodular surface spread across the right side of the scalp. There was focal ulceration and yellow discharge on the mass. (Figure 1-b)

Laboratory examination showed normal blood, urine and stool routine with normal liver and kidney functions. MRI, brain CT, and ultrasonography of the abdomen did not reveal any abnormalities. Three biopsy specimens were obtained from one of the nodules on the face and two different sites of the mass on the scalp. 
Histopathologic examination of the biopsy from the pigmented lesions revealed junctional and dermal melanocytic nests. Nests of nevus cells extend deeply in the dermis and around hair follicles. There were also some melanophages in papillary dermis. Cellular atypia, necrosis and atypical mitoses were not seen in these specimens. ( Figure 2-a,b,c)

Discussion

Melanocytic nevi are benign proliferation of melanocytes arranged in nests. Melanocytic nevi are mostly present at birth although some may develop within two years after birth. (6)

The prevalence of congenital pigmented lesions is estimated about $2.5 \%$ but biopsy-confirmed melanocytic nevi are reported only in $1 \%$ of infants. The prevalence of CMN ranges from 0.005 to $2.7 \%$ depending on the size of the lesion in greatest diameter. (8)

CMN are caused by gain-of-function somatic mutations in either BRAF at V600 or NRAS at Q6. These mutations affect the microtubule-associated protein kinase (MAPK) signal trans-duction pathway, leading to abnormal proliferation of embryonic melanoblasts. These abnormal melanoblasts scatter to various parts of the body, leading to nevi. CMN are predominantly caused by sporadic de novo mutations, although familial cases have been reported. (2)

The clinical presentation varies depending on size, location and age. Based on their size in greatest diameter, CMN are categorized into three groups: small $(<1.5 \mathrm{~cm})$, medium $(1.5-19.9 \mathrm{~cm})$ and large or giant ([?]20 $\mathrm{cm})$. The size of CMN is extremely important since it increases the risk of malignancy transformation and is a decisive factor in cosmetic and therapeutic approaches. (9)

GCMN are mostly characterized as brownish lesions with distinct borders and hypertrichosis. In comparison to acquired melanocytic nevi, CMN have a heterogeneous morphology and they're larger in size. The clinical presentation may vary in different stages of life. At birth they are usually flat and as the child grows they become darker in color, thicker and develope coarse hair. However in our case, the patient was born with dark brown nodules and papules on the trunk and face, rather than flat lesions and associated hypertichosis was absent. GCMN typically appear on trunk $(38 \%)$ and extremities $(38 \%)$. Head and neck lesions are less common $(14 \%)$. $(9,10)$

In histologic examination, epidermal changes of CMN are similar to acquired melanocytic nevi however they cab be distinguished by the presence of nevus cells in the deep dermis and subcutaneous tissue; arrangement of these cells among the collagen bundles of the lower reticular dermis; the involvement of sebaceous glands, blood vessels, erector muscles of hair follicles, eccrine glands and lymphatic vessels. (6)

A major concern about CMN is malignant transformation to melanoma which can develop within the nevus or elsewhere, especially the central nervous system. Size, location, and type of histology are important factors which help to assess the risk of developing melanoma in CMN. The risk increases in larger lesions and for GCMN, the risk of developing melanoma has been reported to be as high as $5-7 \%$ by the age of 60 years. The nevi located on the posterior scalp, neck, back, and buttock are at more risk of developing melanoma than lesions on the face and limbs. $(4,11)$

Malignant transformation occurs in the first decade of life in most of the cases. In our case in histologic examination, there was no evidence of cellular atypia, atypical mitoses or focal necrosis within these three samples.

Another associated complication in congenital melanocytic nevi is neurocutaneous melanosis (NCM) defined as melanocytic proliferation within the leptomeninges and brain parenchyma and is divided into symptomatic and asymptomatic. Symptomatic NCM frequently manifests in the first 2-3 years of life with signs of raised intracranial pressure due to hydrocephalus or mass effect and is accompanied by poor prognosis. The risk of neurocutaneous melanosis is higher in large or giant nevi with a greater number of satellite lesions and a posterior axial location (involving the head, neck, back and buttocks) $(12,13)$. 
Since our patient was at risk for neurocutaneous melanosis and had not been screened for it earlier, MRI imaging of head and spine was requested for the patient that showed no abnormality. Her parents were provided guidance for detecting signs and symptoms of NCM.

The patient was referred to the plastic surgery department for providing tissue expander and serial excision.

Conclusion

Giant congenital melanocytic nevi are caused by benign proliferation of melanocytes which are larger than $20 \mathrm{~cm}$. They can be associated with complications such as neurocutaneous melanoma and there is a risk of malignancy which is higher in larger lesions. Thus, it is important to monitor patients for such conditions and remove the nevi to reduce the cosmetic burden.

\section{Author Contribution:}

Dr. Fatemeh Mohaghegh visited and examined the patient and formed diagnostic and therapeutic plans for the patient and has also been involved in drafting the manuscript and revising it critically for important intellectual content.

Dr. Ali Asilian revised the manuscript and gave the final approval of the version to be published.

Dr. Samin Nabavinejad has been involved in collecting information from the patient including the pictures and preparing the biopsy specimen. She also participated in drafting the manuscript.

Dr. Mina Rezaei participated in writing the manuscript and collecting information from the patient.

\section{References}

1. Shetty J, Bhat SP, Sathishchandra BK, Shetty V. Giant Congenital Melanocytic Nevus of Scalp, Mimicking Turban Tumor. RGUHS Journal of Medical Sciences. 2011;1(2):45-7.

2. Etchevers HC. Hiding in plain sight: molecular genetics applied to giant congenital melanocytic nevi. Journal of Investigative Dermatology. 2014;134(4):879-82.

3. Kopf AW, Bart RS, Hennessey P. Congenital nevocytic nevi and malignant melanomas. Journal of the American Academy of Dermatology. 1979;1(2):123-30.

4. Krishnamurthy S, Kavita GU, Ali SM, Parvatappa N. Giant Congenital Cerebriform Melanocytic Nevus of the Scalp in Adult. Journal of Interdisciplinary Histopathology. 2014;2(2):108-11.

5. Meshram GG, Kaur N, Hura KS. Giant Congenital Melanocytic Nevi: An Update and Emerging Therapies. Case reports in dermatology. 2018;10(1):24-8.

6. Viana ACL, Gontijo B, Bittencourt FV. Giant congenital melanocytic nevus. Anais brasileiros de dermatologia. 2013;88(6):863-78.

7. Tannous ZS, Mihm Jr MC, Sober AJ, Duncan LM. Congenital melanocytic nevi: clinical and histopathologic features, risk of melanoma, and clinical management. Journal of the American Academy of Dermatology. 2005;52(2):197-203.

8. Nikfarjam J, Chambers E. Congenital melanocytic nevi and the risk of malignant melanoma: establishing a guideline for primary-care physicians. Einstein Journal of Biology and Medicine. 2016;27(2):59-66.

9. Bhagyalakshmi A, Sudhakar P, Uma P, Devi U. Giant congenital melanocytic nevus scalp: report of a rare case. Int J Res Med Sci. 2013;1(3):317.

10. Alikhan A, Ibrahimi OA, Eisen DB. Congenital melanocytic nevi: where are we now?: part I. Clinical presentation, epidemiology, pathogenesis, histology, malignant transformation, and neurocutaneous melanosis. Journal of the American Academy of Dermatology. 2012;67(4):495-e1.

11. Kaplan EN. Congenital nevi-risk of malignancy. The Western journal of medicine. 1989;150(1):78-9. 
12. Kinsler VA, Thomas AC, Ishida M, Bulstrode NW, Loughlin S, Hing S, et al. Multiple congenital melanocytic nevi and neurocutaneous melanosis are caused by postzygotic mutations in codon 61 of NRAS. Journal of Investigative Dermatology. 2013;133(9):2229-36.

13. DeDavid M, Orlow SJ, Provost N, Marghoob AA, Rao BK, Wasti Q, et al. Neurocutaneous melanosis: clinical features of large congenital melanocytic nevi in patients with manifest central nervous system melanosis. Journal of the American Academy of Dermatology. 1996;35(4):529-3

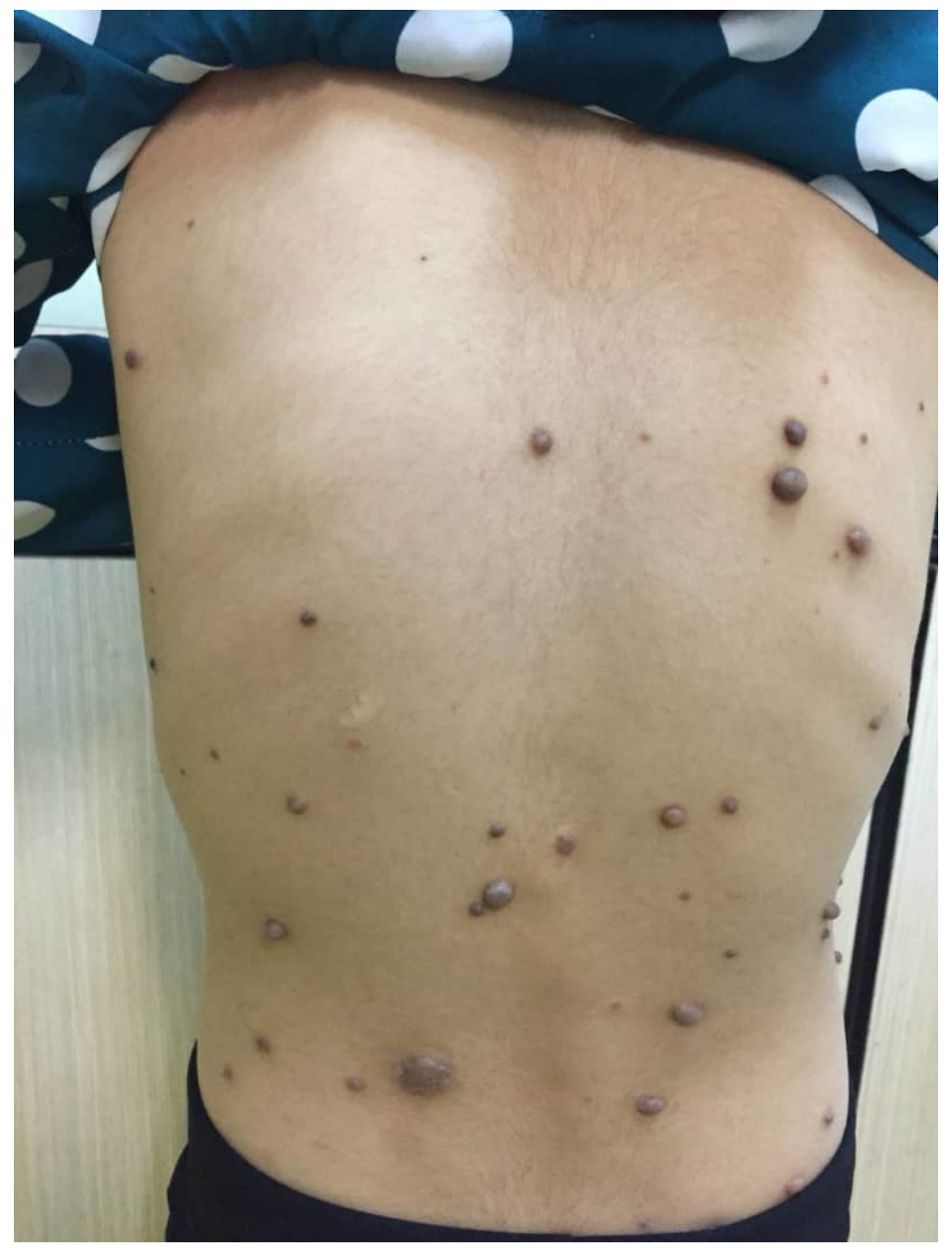




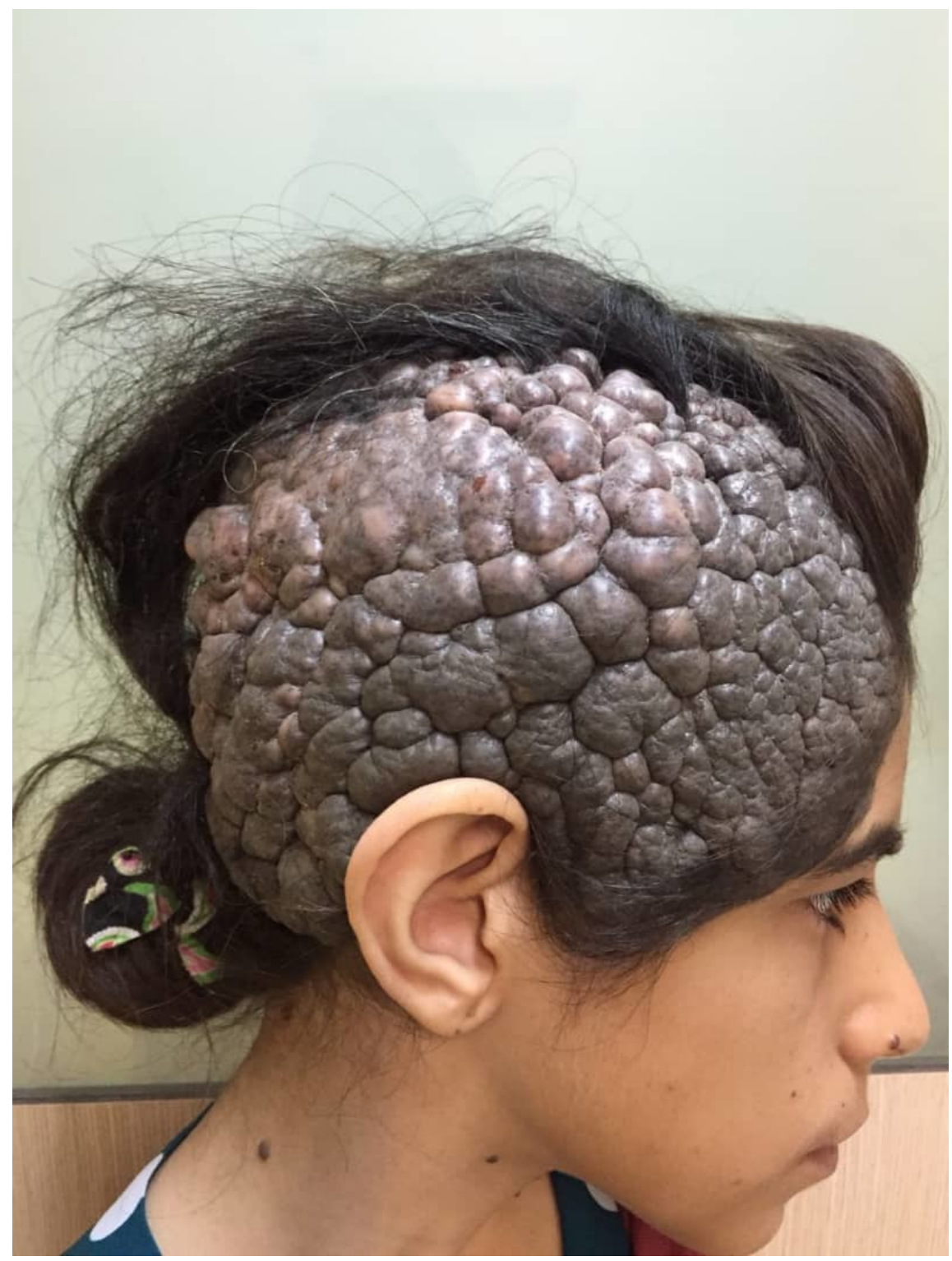



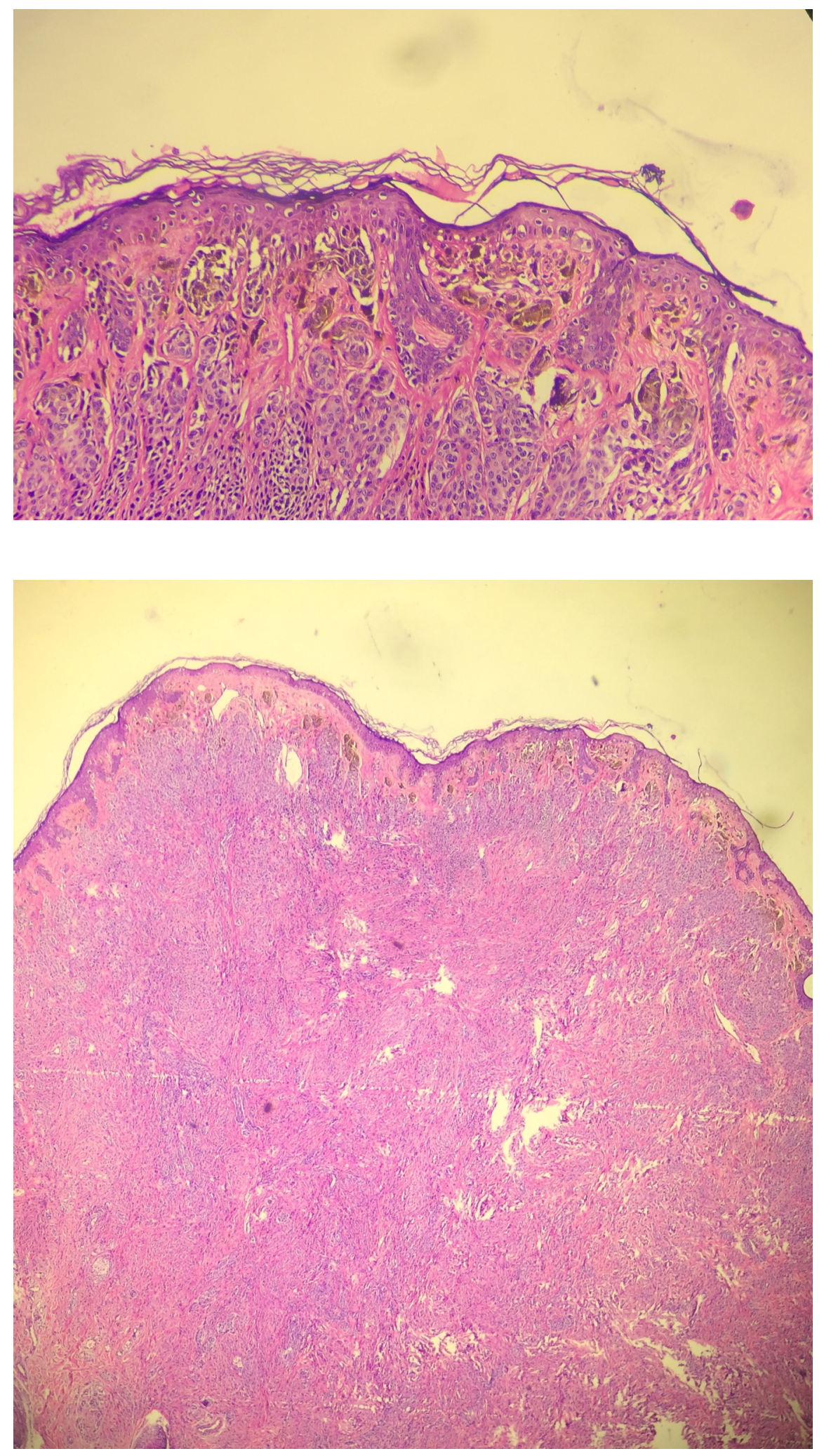


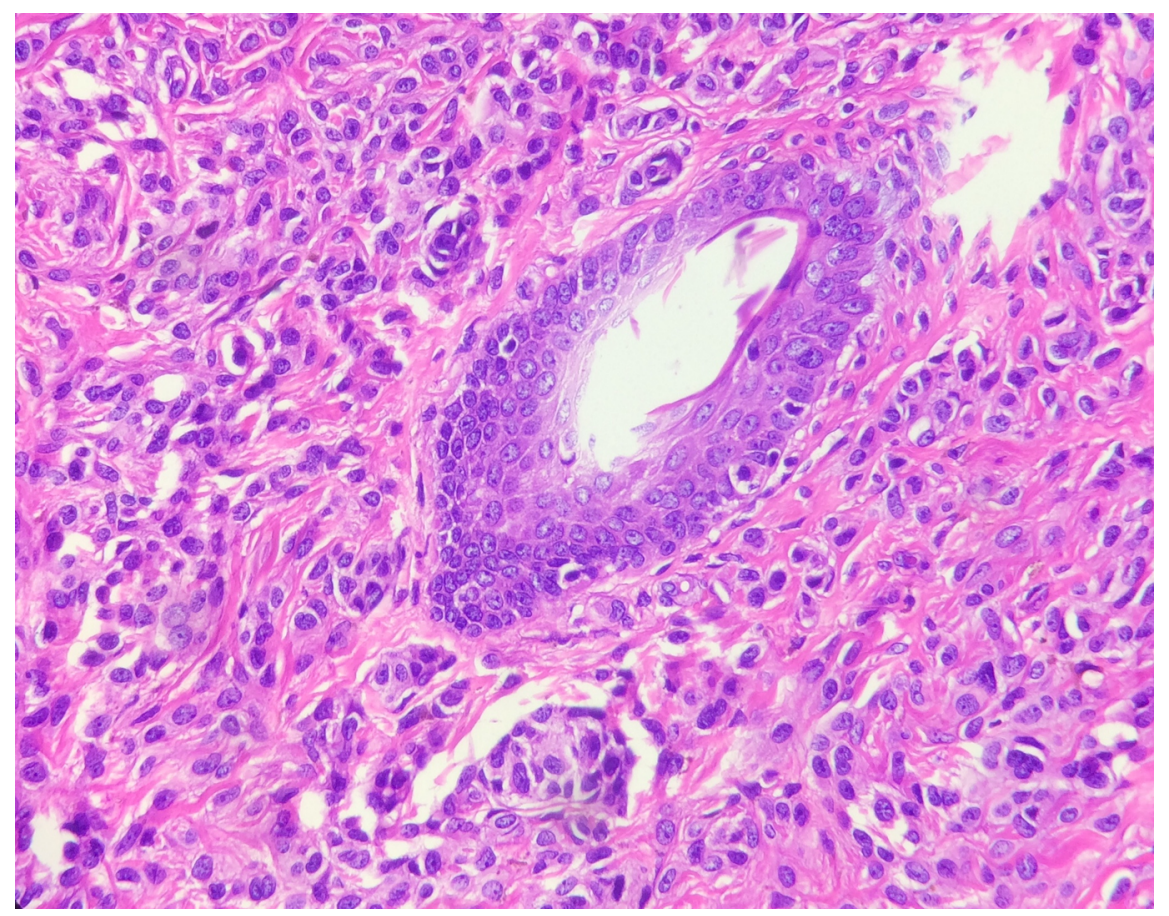

